\title{
In Vitro Validation and Clinical Testing of an Indirect Calorimetry System for Ventilated Preterm Infants That Is Unaffected by Endotracheal Tube Leaks and Can Be Used during Nasal Continuous Positive Airway Pressure
}

\author{
KARL BAUER, JÖRG KETTELER, MAREN LAURENZ, AND HANS VERSMOLD
}

Department of Pediatrics, Freie Universität Berlin, Universitätsklinikum Benjamin Franklin, 12200

Berlin, Germany

\begin{abstract}
ABSTR
Energy expenditure measurements in ventilated preterm in-
fants are difficult because indirect calorimetry underestimates
energy expenditure during gas leaks around uncuffed endotra-
cheal tubes routinely used in preterm infants or during nasal
continuous positive airway pressure (CPAP). We, therefore,
developed a breath collector that simultaneously sampled expired
air expelled at the ventilator outlet and escaping via the tube leak
from the infant's mouth and nose. Our breath collector was
combined with a proprietary calorimeter (Deltatrac II). In vitro
validation was done by methanol burning ( $\mathrm{Vo}_{2}, 13.8 \mathrm{~mL} / \mathrm{min}$;
Vco ${ }_{2}, 9.2 \mathrm{~mL} /$ min) during intermittent positive pressure ventila-
tion (IPPV) with two commonly used ventilators $(\mathrm{Sechrist} \mathrm{IV-}$
$100 \mathrm{~B}$ and Infant Star). Measurement error was determined at
different ventilator flows, peak inspiratory pressures of $12-24 \mathrm{~cm}$
$\mathrm{H}_{2} \mathrm{O}$, and during a complete tube leak. The mean measurement
error with both ventilators was low (Vo $\mathrm{O}_{2} \pm 3 \%, \mathrm{Vco}_{2} \pm 2 \%$ )
even during a complete tube leak and did not increase with peak
inspiratory pressure. The system response time was 2 min. In
\end{abstract}
Nutritional intake has to meet energy requirements. Although the EE of spontaneously breathing growing preterm infants is well documented $(1,2)$, there is a great need for EE measurements in critically ill and ventilated preterm infants (3). These infants can have large variations in EE due to the underlying disease, surgery, therapeutic interventions, or medication. The reason for the paucity of data is the methodologic difficulties for indirect calorimetry in ventilated preterm infants. The routine use of uncuffed endotracheal tubes leads to unpredictable leakage of expired air through mouth and nose (tube leak) in up to $50 \%$ of ventilated neonates (4), and the

Received April 26, 2000; accepted October 20, 2000.

Correspondence and reprint requests: Karl Bauer, M.D., Department of Pediatrics, Freie Universität Berlin, Universitätsklinikum Benjamin Franklin, 12200 Berlin, Germany. vivo measurements at the bedside were performed in 25 preterm infants (body weight, 537-1402 g). Energy expenditure during IPPV was $40 \pm 9 \mathrm{kcal} / \mathrm{kg}$ per day and $46 \pm 15 \mathrm{kcal} / \mathrm{kg}$ per day during nasal CPAP. The tube leak in the preterm infants studied during IPPV was 0 to $47 \%$, and during nasal CPAP 84 to $97 \%$. In conclusion, indirect calorimetry performed with our breath collector was accurate during IPPV and nasal CPAP and was unaffected by tube leaks. (Pediatr Res 49: 394-401, 2001)
Abbreviations
CPAP, continuous positive airway pressure
EE, energy expenditure
Fio $_{2}$, fraction of inspiratory oxygen
PEEP, positive endexpiratory pressure
PIP, peak inspiratory pressure
$\mathbf{R Q}$, respiratory quotient
$\mathrm{Vco}_{2}$, carbon dioxide production
$\mathbf{V o}_{2}$, oxygen consumption

commonly used nasal CPAP represents a maximal tube leak. In previous measurements of $\mathrm{EE}$, many ventilated preterm infants with tube leaks had to be excluded from analysis $(5,6)$, and no measurements during nasal CPAP have been reported.

We, therefore, developed an indirect calorimetry system with the following capabilities: 1) accurate measurements in intubated preterm infants regardless of tube leak, 2) accurate measurements during nasal CPAP, 3) applicable in combination with pressure-controlled constant-flow ventilators, 4) applicable during increased $\mathrm{Fio}_{2}, 5$ ) accurate measurements of low absolute values of $\mathrm{Vo}_{2}$ and $\mathrm{Vco}_{2}$, and 6 ) easy to use at the bedside without undue disturbance of the patient.

The purpose of the present study was to validate this calorimetry system in vitro and to evaluate its performance in the clinical setting. 


\section{METHODS}

\section{The Breath Collector}

We built a breath collector that simultaneously sampled expired air expelled from the ventilator and coming from the infant's mouth and nose (Fig. 1). The breath collector was made from a $200-\mathrm{mL}$ plastic container (Sarstedt, Nürnberg, Germany). Air from the infant's mouth and nose entered through the open container bottom $(\varnothing 4 \mathrm{~cm})$. A small indentation in the lower sidewall was for the endotracheal tube. In the container lid was an opening with a tube leading to the calorimeter.
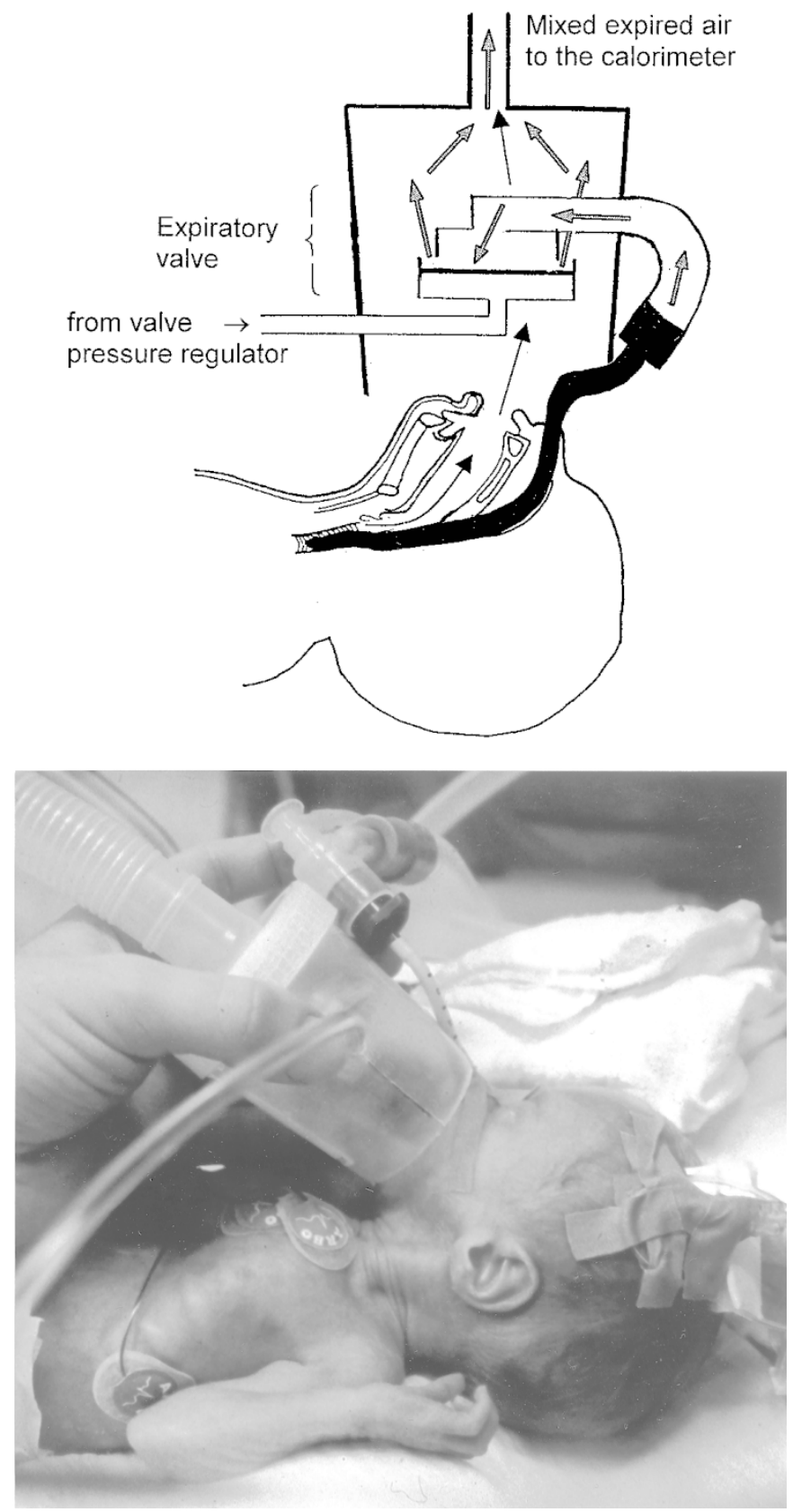

Figure 1. Use of the breath collector in combination with ventilator A. (Top) Schematic drawing of the measurement setup. Thin arrows represent flow of exhaled gas escaping via the tube leak; gray arrows represent exhaled gas from the endotracheal tube. (Bottom) Measurement in a preterm infant.

\section{Calorimeter}

We used the breath collector together with the commercially available indirect calorimeter Deltatrac II (Datex Division Instrumentarium Corp., Helsinki, Finland). A constant-flow generator in the calorimeter withdraws the expired air diluted with room air from the breath collector. The flow rate is independent of the added flux of room air. $\mathrm{CO}_{2}$ and $\mathrm{Vo}_{2}$ are calculated from the constant flow of the diluted expired air and the concentration differences between diluted expired air and room air. This requires high-resolution gas sensors that can measure small concentration differences. The paramagnetic oxygen sensor and the infrared $\mathrm{CO}_{2}$ sensor in the Deltatrac II have a high resolution of $0.01 \%$ (7). We modified the constantflow generator to produce flow rates between 1 and $10 \mathrm{~L} / \mathrm{min}$ and calibrated the flow generator by quantitative methanol burning (8).

\section{Components of the In Vitro Validation System}

We tested the breath collector in combination with different ventilators and at different ventilator settings with a self-made in vitro validation system. It consisted of an EE simulation by quantitative methanol burning, a test lung, and the breath collector/calorimeter (Fig. 2). We simulated EE with a miniburner previously built to validate indirect calorimetry in spontaneously breathing infants (8). In short, methanol (methanol extra pure $>99.5 \%$; Merck, Darmstadt, Germany) delivered by a precision infusion pump (Perfusor fm; BraunMelsungen AG, Melsungen, Germany) at a rate of $1 \mathrm{~mL} / \mathrm{min}$ was burned at the tip of a glass capillary, producing a $\mathrm{Vco}_{2}$ of $9.2 \mathrm{~mL} / \mathrm{min}$ and $\mathrm{a} \mathrm{Vo}_{2}$ of $13.8 \mathrm{~mL} / \mathrm{min}(=\mathrm{EE}$ of $0.16 \mathrm{kcal} / \mathrm{min}$ or $0.69 \mathrm{~kJ} / \mathrm{min}$ ). These $\mathrm{Vo}_{2}$ and $\mathrm{Vco}_{2}$ values are in the range expected in an infant of approximately $2 \mathrm{~kg}$ body weight. Methanol burning was done inside a small airtight box incorporated in the ventilator circuit. We used a plastic test lung with a volume of $40 \mathrm{~mL}$ (Draeger AG, Lübeck, Germany).

\section{Protocol for the In Vitro Experiments}

We tested the breath collector in combination with two pressure-controlled constant-flow ventilators for preterm infants, the Sechrist IV-100B (Sechrist Industries, Anaheim, CA, U.S.A.) (ventilator A) and the Infant Star software version 83 (Nellcor Puritan Bennet Inc., Carlsbad, CA, U.S.A.) (ventilator B). When used with ventilator A, the expiratory valve located at the end of the ventilator circuit was placed inside the breath collector with the gas outlets of the valve pointing toward the calorimeter to facilitate gas sampling (Fig. 2A). In ventilator B, the expiratory valve is mounted on the ventilator. We sealed this expiratory block with adhesive tape to prevent any gas leakage and directed the gas flow from the valve via a plastic tube to the breath collector (Fig. $2 B$ ).

First, the gas analyzers were calibrated with a known gas mixture $\left(5 \% \mathrm{CO}_{2}\right.$ and $95 \% \mathrm{O}_{2}$, Datex Division Instrumentarium Corp., Helsinki, Finland), and the calorimeter flow was set to $6 \mathrm{~L} / \mathrm{min}$. Then the ventilator was switched on, the methanol flame in the ventilator circuit was lit, and the burning chamber was closed airtight. All experiments were done in room air 

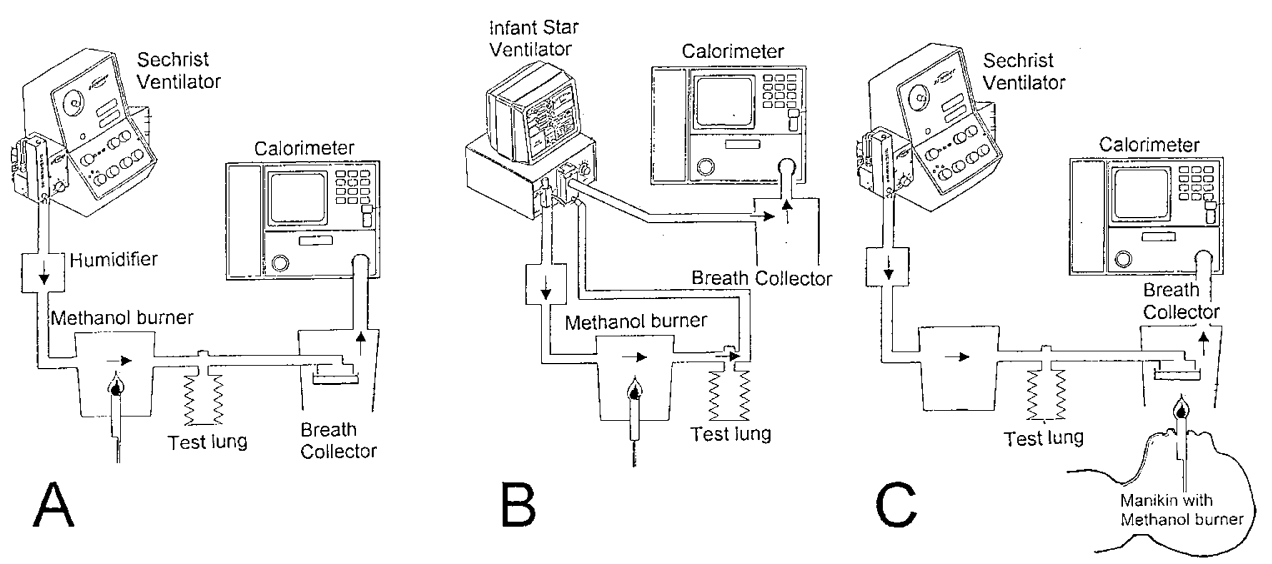

Figure 2. Schematic drawing of the in vitro validation setup with ventilator A $(A)$, ventilator B $(B)$, and during simulation of a complete tube leak $(C)$. Arrows indicate the direction of gas flow in the ventilator circuits.

because of the danger of explosion during combustions at raised $\mathrm{Fio}_{2}$. Gases from the ventilator passed through the burning chamber and test lung to the breath collector that was held several millimeters above a flat surface by a research assistant to mimic the application in an infant. To avoid contaminations by expired air from the research assistant, he stayed at least $30 \mathrm{~cm}$ away from the breath collector during measurements.

We determined the measurement error for the combination with each ventilator at ventilator settings commonly used for preterm infants, i.e. PIP up to $24 \mathrm{~cm} \mathrm{H}_{2} 0$, a PEEP of $3 \mathrm{~cm} \mathrm{H}_{2} 0$, and a rate of $60 / \mathrm{min}$. For each experimental condition, we performed five separate measurements. The actual measurement, lasting $20 \mathrm{~min}$, followed $10 \mathrm{~min}$ of equilibration. The measurement error was calculated as

$$
\text { measurement error }=\frac{\text { observed value }- \text { expected value }}{\text { expected value }} \times 100
$$

Determination of measurement error at different ventilator flows. We determined the measurement error at a calorimeter flow of $6 \mathrm{~L} / \mathrm{min}$ at constant ventilator flows of 3, 4, 5, and 6 $\mathrm{L} / \mathrm{min}$. The other ventilator settings were kept constant (rate, 60/min; PEEP, $3 \mathrm{~cm} \mathrm{H} \mathrm{H}_{2} \mathrm{O}$; PIP, $15 \mathrm{~cm} \mathrm{H}_{2} \mathrm{O}$ ).

Determination of measurement error at different PIP. We changed PIP from 12 to $24 \mathrm{~cm} \mathrm{H}_{2} \mathrm{O}$. The other ventilator settings were kept constant (rate, 60/min; PEEP, $3 \mathrm{~cm} \mathrm{H}_{2} \mathrm{O}$; flow, $4 \mathrm{~L} / \mathrm{min}$ ).

Determination of measurement error with a simulated complete tube leak. To simulate a complete tube leak, i.e. all expired air comes out of the ventilated infant's mouth and nose, the methanol burner was placed inside a manikin the size of a preterm infant with the methanol flame burning at the position of the manikin's mouth. The manikin is described in detail elsewhere (8). Gas was sampled by holding the breath collector with the bottom opening above the manikin's face while mechanical ventilation was simulated at a rate of $60 / \mathrm{min}$, PIP of $15 \mathrm{~cm} \mathrm{H}_{2} \mathrm{O}$, PEEP of $3 \mathrm{~cm} \mathrm{H}_{2} \mathrm{O}$ (Fig. 2C).

Measurement of response time. The response time of the system was tested by measuring the time interval between a step change in methanol combustion rate from 1.0 to 1.1 $\mathrm{mL} / \mathrm{min}$ (= increase of $\mathrm{Vco}_{2}$ from 9.2 to $10.1 \mathrm{~mL} / \mathrm{min}$ ) and the accurate and stable measurement of the increased $\mathrm{VcO}_{2}$.

\section{Clinical Testing}

We measured $\mathrm{Vo}_{2}$ and $\mathrm{Vco}_{2}$ in mechanically ventilated preterm infants and infants receiving nasal CPAP. To expose our system to a variety of clinical situations, we studied patients of different sizes, postnatal ages, and intensity of mechanical ventilation. Exclusion criteria were the following: 1) no parental consent, 2) unstable mechanical ventilation, 3) birth weight $\left.<500 \mathrm{~g}, 4) \mathrm{PIP}>25 \mathrm{~cm} \mathrm{H}_{2} \mathrm{O}, 5\right) \mathrm{Fio}_{2}>0.6 \mathrm{~L} / \mathrm{L}$. All infants were in incubators (model 8000, Draeger AG, Lübeck, Germany) at thermoneutral temperature. Constant flow at the ventilators was $4 \mathrm{~L} / \mathrm{min}$; the internal calorimeter flow was $6 \mathrm{~L} / \mathrm{min}$. Nasal CPAP was provided via a shortened endotracheal tube placed in the epipharynx.

The present study was approved by the Institutional Review Board of the hospital, and written parental consent was obtained.

\section{Measurement Protocol for Mechanically Ventilated Infants and Infants on Nasal CPAP}

After the infant had received nursing care and gavage feeding, he was positioned supine and the breath collector was connected to the ventilator circuit. The breath collector was easy to connect to the ventilator circuits. This required a short disconnection of the expiratory valve of ventilator A for one or two breaths. No disconnection was necessary when the breath collector was used with ventilator B. Measurements started after an equilibration period of $10 \mathrm{~min}$.

Measurement of tube leak. To measure the tube leak, the breath collector was placed beside the infant's head for $30 \mathrm{~min}$ so that the air escaping through mouth and nose was no longer collected. The tube leak was calculated from the difference in $\mathrm{VcO}_{2}$ measurements with and without collecting gas from the infant's mouth and nose.

Measurement of $\mathrm{Vo}_{2}$ and $\mathrm{Vco}_{2}$. To simultaneously collect expired air from the ventilator valve and from the tube leak, the 
breath collector was held several millimeters above the infant's mouth and nose by a research assistant without actually touching the infant. When the infant turned the head, the research assistant repositioned the breath collector to keep it in front of mouth and nose (Fig. 1).

In infants on nasal CPAP breathing room air, $\mathrm{Vco}_{2}$ and $\mathrm{Vo}_{2}$ were continuously measured for $120 \mathrm{~min}$.

In endotracheally intubated infants with a raised $\mathrm{Fio}_{2}, \mathrm{Vco}_{2}$ was measured continuously for $90 \mathrm{~min}$. The $\mathrm{Vo}_{2}$ could not be measured due to the dilution with room air. Instead, we measured the RQ for $30 \mathrm{~min}$. The RQ, the $\mathrm{Vco}_{2} / \mathrm{Vo}_{2}$ ratio, is unaffected by partial loss of expired air and is accurate in raised $\mathrm{FiO}_{2}$ as long as no room air dilutes the collected gas. For the duration of the RQ measurement, therefore, we reduced the calorimeter flow to $1 \mathrm{~L} / \mathrm{min}$ at an unchanged ventilator flow of $4 \mathrm{~L} / \mathrm{min}$. Thus, much more gas entered the breath collector than was withdrawn. This spillover prevented the dilution of the collected gas by room air. As an additional precaution against dilution by room air, the breath collector was wrapped in a thin polyethylene foil. During RQ measurements, oxygen was taken from a gas tank instead of the wall source to minimize $\mathrm{Fio}_{2}$ fluctuations.

In infants on nasal CPAP in raised $\mathrm{Fio}_{2}$, only $\mathrm{Vco}_{2}$ was measured because the amount of expired air from the expiratory valve was insufficient to measure the RQ.

Transcutaneous $\mathrm{PCO}_{2}$ and heart rate were measured continuously, and the infant's activity was scored every minute by the research assistant using a modified Brueck score (9). Time spent in sleep was defined as the time with a Brueck score of -3 (eyes closed, only facial movements) or -4 (eyes closed, no movements).

\section{Calculations}

$\mathrm{Vo}_{2}(\mathrm{~mL} / \mathrm{min}), \mathrm{Vco}_{2}(\mathrm{~mL} / \mathrm{min})$, and RQ were calculated from the $\mathrm{O}_{2}$ and $\mathrm{CO}_{2}$ concentrations measured in the inspiratory gas $\left(\mathrm{FiO}_{2}\right.$ and $\left.\mathrm{FicO}_{2}\right)$ and in the diluted expiratory gas passing through the calorimeter $\left(\mathrm{FeO}_{2}\right.$ and $\left.\mathrm{FecO}_{2}\right)$ and the constant internal calorimeter flow $(\mathrm{Q} ; \mathrm{mL} / \mathrm{min})$ by using the Haldane transformation (Equations 2-4) (10). In infants with raised $\mathrm{Fio}_{2}$, we measured $\mathrm{Vco}_{2}$ and $\mathrm{RQ}$ and calculated $\mathrm{Vo}_{2}$ according to Equation 5.

$$
\begin{gathered}
\mathrm{VCO}_{2}=\left(\mathrm{F}_{\mathrm{e}} \mathrm{CO}_{2}-\mathrm{F}_{\mathrm{i}} \mathrm{CO}_{2}\right) \times \mathrm{Q} \\
\mathrm{RQ}=\frac{1-\mathrm{F}_{\mathrm{i}} \mathrm{O}_{2}}{\left[\left(\mathrm{~F}_{\mathrm{i} \mathrm{O}_{2}}-\mathrm{F}_{\mathrm{e}} \mathrm{O}_{2}\right) / \mathrm{F}_{\mathrm{e}} \mathrm{CO}_{2}\right]-\mathrm{F}_{\mathrm{i}} \mathrm{O}_{2}} \\
\mathrm{VO}_{2}=\frac{\left(\mathrm{F}_{\mathrm{i}} \mathrm{O}_{2}-\mathrm{F}_{\mathrm{e}} \mathrm{O}_{2}\right)-\mathrm{F}_{\mathrm{i}} \mathrm{O}_{2} \times \mathrm{F}_{\mathrm{e}} \mathrm{CO}_{2}}{1-\mathrm{F}_{\mathrm{i}} \mathrm{O}_{2}} \times Q \\
\mathrm{VO}_{2}=\mathrm{RQ} / \mathrm{VCO}_{2}
\end{gathered}
$$

EE ( $\mathrm{kcal} / \mathrm{d})$ was calculated from $\mathrm{Vo}_{2}, \mathrm{Vco}_{2}$, and urinary nitrogen excretion (Un) that was estimated as $0.15 \mathrm{~g} \mathrm{~N}$ per $\mathrm{kg}$ body weight (11).

$$
\mathrm{EE}=5.5 \times \mathrm{VO}_{2}+1.76 \times \mathrm{VCO}_{2}-1.99 \times \mathrm{Un}
$$

\section{Statistical Analysis}

Data were analyzed with the SPSS statistical software (SPSS Inc., Chicago, IL, U.S.A.). Normally distributed data are presented as mean $\pm \mathrm{SD}$, not normally distributed data as median (range).

\section{RESULTS}

\section{In Vitro Experiments}

Determination of measurement error at different ventilator flows. As long as the calorimeter flow was higher than the flow at the ventilator, gas from the methanol burn was completely sampled and $\mathrm{Vo}_{2}$ and $\mathrm{Vco}_{2}$ measurements were sufficiently close to the predicted values to be clinically acceptable (Fig. 3). The measurement error was small at a ventilator flow of 4 $\mathrm{L} / \mathrm{min}$ and a calorimeter flow of $6 \mathrm{~L} / \mathrm{min}$ in both ventilator types [ventilator $\mathrm{A}, 1 \pm 1 \%\left(\mathrm{Vco}_{2}\right)$ and $-1 \pm 5 \%\left(\mathrm{Vo}_{2}\right)$; ventilator $\mathrm{B}, 3 \pm 2 \%\left(\mathrm{Vco}_{2}\right)$ and $\left.-2 \pm 1 \%\left(\mathrm{Vo}_{2}\right)\right]$. Therefore, this flow combination was used in all subsequent experiments.

Determination of measurement error at different PIP. Changing PIP between 12 and $24 \mathrm{~cm} \mathrm{H}_{2} \mathrm{O}$ did not influence the $\mathrm{Vo}_{2}$ and $\mathrm{Vco}_{2}$ measurement error with both ventilators (Table 1).

Determination of measurement error with a simulated complete tube leak. When methanol was burned with the manikin simulating a complete tube leak during mechanical ventilation, measurement error with ventilator $A$ was $1 \pm 4 \%$ $\left(\mathrm{Vco}_{2}\right)$ and $0.3 \pm 4 \%\left(\mathrm{Vo}_{2}\right)$, and measurement error with ventilator $\mathrm{B}$ was $1 \pm 2 \%\left(\mathrm{Vco}_{2}\right)$ and $-0.5 \pm 3 \%\left(\mathrm{Vo}_{2}\right)$.

Measurement of the response time. The response time of the system to a step change in methanol burn was $2 \mathrm{~min}$ (Fig. 4).

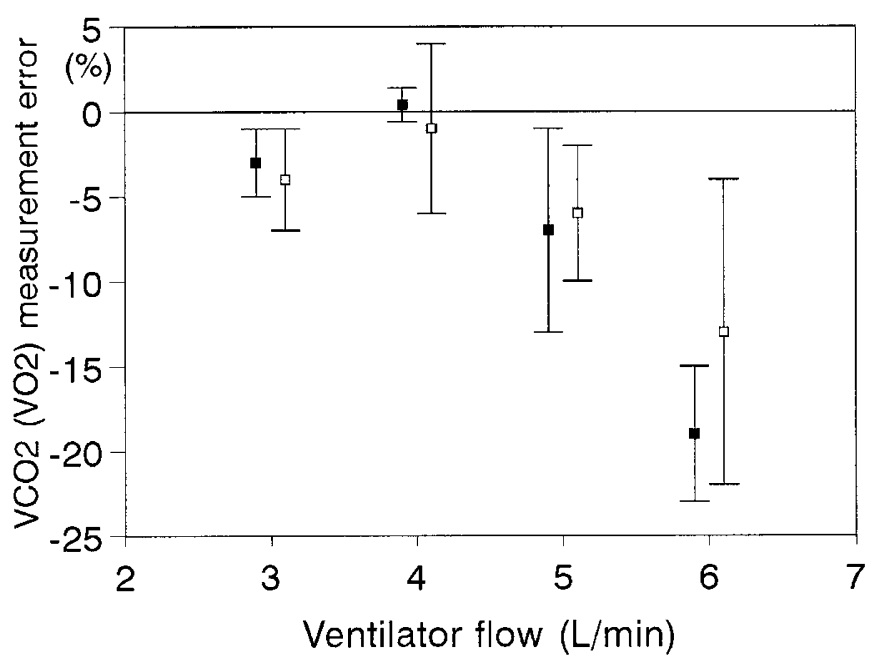

Figure 3. Measurement error for $\mathrm{Vo}_{2}$ (open squares) and $\mathrm{Vco}_{2}$ (closed squares) at different ventilator flow rates (ventilator $\mathrm{A}$ ) during quantitative methanol burning. The calorimeter flow rate was $6 \mathrm{~L} / \mathrm{min}$. Data are mean $\pm \mathrm{SD}$ of five methanol burns. 
Table 1. $\mathrm{VCO}_{2}$ and $\mathrm{VO}_{2}$ measurement error at different PIP with ventilator $A$ and ventilator $B$

\begin{tabular}{ccccr}
\hline & \multicolumn{2}{c}{$\mathrm{VCO}_{2}$ measurement error (\%) } & \multicolumn{2}{c}{$\mathrm{Vo}_{2}$ measurement error $(\%)$} \\
\cline { 2 - 4 } PIP & ventilator A & ventilator B & ventilator A & ventilator B \\
\hline $12 \mathrm{~cm} \mathrm{H}_{2} \mathrm{O}$ & $1 \pm 1$ & $1 \pm 4$ & $-1 \pm 5$ & $2 \pm 5$ \\
$20 \mathrm{~cm} \mathrm{H}_{2} \mathrm{O}$ & $0 \pm 2$ & $0 \pm 2$ & $-2 \pm 5$ & $-2 \pm 1$ \\
$24 \mathrm{~cm} \mathrm{H}_{2} \mathrm{O}$ & $-1 \pm 1$ & n.d. & $-2 \pm 4$ & n.d. \\
\hline
\end{tabular}

n.d., not done. Values are mean $\pm \mathrm{SD}$ of five experiments each.

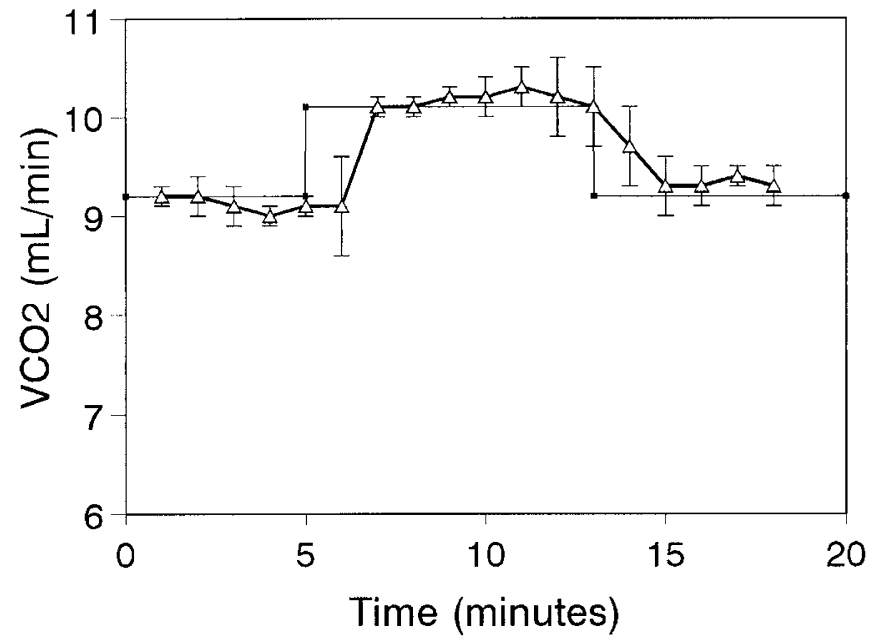

Figure 4. Response time of the system. A step change in $\mathrm{Vco}_{2}$ from 9.2 to $10.1 \mathrm{~mL} / \mathrm{min}$ was produced by increasing methanol delivery rate. Data (triangles) are mean $\pm \mathrm{SD}$ of five methanol burns.

\section{Clinical Testing}

We studied 15 preterm infants during intermittent positive pressure ventilation and 10 preterm infants during nasal CPAP (ventilator A) (Table 2). The infants were not disturbed by the measurement and were sleeping most (median $\geq 85 \%$ ) of the observation period.

Measurement of tube leak. The tube leaks in the intubated preterm infants varied considerably from 0 to $47 \%$ (Table 3). Tube-leak size was independent of PIP or body weight. During nasal CPAP, most of the expired air escaped via nose and mouth, and the tube leak ranged from 84 to $97 \%$.

Measurement of $\boldsymbol{V C o}_{2}$ and $\mathrm{Vo}_{2}$. For these measurements, air escaping from the expiratory valve and via mouth and nose were sampled simultaneously and the values are, therefore, undiminished by tube leaks (Table 4). $\mathrm{Vco}_{2}$ was measured in all infants. Transcutaneously measured $\mathrm{PcO}_{2}\left(\mathrm{Tcpco}_{2}\right)$ was not different at start and end of the measurements, indicating that no $\mathrm{CO}_{2}$ retention had occurred. $\mathrm{Vo}_{2}$ was measured in seven infants on nasal CPAP breathing room air. $\mathrm{Vo}_{2}$ was calculated from $\mathrm{VcO}_{2}$ and $\mathrm{RQ}$ in all endotracheally intubated preterm infants with raised $\mathrm{Fio}_{2}$. In three infants on nasal CPAP at raised $\mathrm{Fio}_{2}$, we could only measure $\mathrm{Vco}_{2}$.

\section{DISCUSSION}

EE measurements in critically ill ventilated neonates are needed to define energy requirements but have been impeded by methodologic problems caused by the strategies of mechanical ventilation typically used in neonates. We developed a breath collector for precise indirect calorimetry that can be used in intubated preterm infants regardless of tube leak and in preterm infants with nasal CPAP. The system is easy to use at the bedside without disturbing the infant.

\section{The Calorimeter}

We used a commercially available calorimeter producing a constant internal gas flow instead of measuring flow rates. Most important in the context of this study, a calorimeter with a constant internal gas flow allows simultaneous sampling of gas from ventilator and infant at the bedside in the same incubator that is used for clinical care without additional complicated equipment. Calorimeters using flow sensors instead of producing a constant flow have been used in mechanically ventilated preterm infants (12-14). But continuous measurements of gas flows are extremely difficult under clinical conditions (15), and a simultaneous collection of gas from ventilator and infant is not possible with these calorimeters.

When a constant-flow calorimeter is used, the dilution of expired air gets higher when the infants studied get smaller. Due to the high-resolution gas analysers we used, we could measure absolute $\mathrm{Vo}_{2}$ or $\mathrm{Vco}_{2}$ as low as $1.5 \mathrm{~mL} / \mathrm{min}$, corresponding to an infant with a body weight of $500 \mathrm{~g}$ at the calorimeter flow of $6 \mathrm{~L} / \mathrm{min}$. We do not recommend use of our system for infants with a body weight $<500 \mathrm{~g}$.

Table 2. Clinical characteristics of the preterm neonates studied

\begin{tabular}{lccc}
\hline & Ventilator A & Ventilator B & Ventilator A \\
& IPPV & IPPV & 10 \\
No. & 10 & 5 & $27(26-29)$ \\
Gestational age (wk) & $26(23-29)^{*}$ & $27(26-28)$ & $932(670-1475)$ \\
Birth weight (g) & $783(554-1244)$ & $784(733-1244)$ & $7(1-21) 5$ \\
Postnatal age (d) & $3(1-5)$ & $5(1-36)$ & $933(687-1402)$ \\
Actual body weight (g) & $732(537-1280)$ & $750(691-1134)$ & $148(141-164)$ \\
Heart rate (per min) & $152(140-163)$ & $160(145-170)$ & $88(70-92)$ \\
Sleep (quiet + active) & $85(78-91)$ & $85(75-94)$ & \\
(\% of total) & & & \\
\hline
\end{tabular}

\footnotetext{
* median (range); IPPV indicates intermittent positive pressure ventilation.
} 
Table 3. Tube leak in preterm neonates during IPPV with two different ventilators and during nasal CPAP

\begin{tabular}{|c|c|c|c|}
\hline & IPPV ventilator A & IPPV ventilator B & $\begin{array}{c}\text { Nasal CPAP } \\
\text { ventilator A }\end{array}$ \\
\hline No. of subjects & 10 & 5 & 10 \\
\hline $\mathrm{F}_{\mathrm{i}} \mathrm{O}_{2}(\mathrm{~L} / \mathrm{L})$ & $0.37(0.25-0.60)^{*}$ & $0.34(0.30-0.50)$ & $0.21(0.21-0.42)$ \\
\hline PIP $\left(\mathrm{cm} \mathrm{H}_{2} \mathrm{O}\right)$ & $14(10-22)$ & $14(11-20)$ & \\
\hline Ventilator rate (/min) & $69(38-79)$ & $60(30-69)$ & \\
\hline Tube leak (\%) & $26(4-47)$ & $5(0-31)$ & $90(84-97)$ \\
\hline
\end{tabular}

Tube leak was calculated from the difference in $\mathrm{VCO}_{2}$ measurements with and without collecting gas from the infant's mouth and nose.

* median (range).

Table 4. $\mathrm{VCO}_{2}, \mathrm{VO}_{2}$, and EE in preterm neonates during IPPV with two different ventilators and during nasal CPAP

\begin{tabular}{|c|c|c|c|}
\hline & $\begin{array}{c}\text { IPPV } \\
\text { ventilator A }\end{array}$ & $\begin{array}{c}\text { IPPV } \\
\text { ventilator B }\end{array}$ & $\begin{array}{l}\text { Nasal CPAP } \\
\text { ventilator A }\end{array}$ \\
\hline No. of subjects & 10 & 5 & 10 \\
\hline $\mathrm{VCO}_{2}(\mathrm{~mL} / \mathrm{kg}$ per min $)$ & $4.8 \pm 0.9 \S$ & $5.8 \pm 1.3$ & $5.2 \pm 1.8$ \\
\hline $\mathrm{TcpCO}_{2}$ end $(\mathrm{mm} \mathrm{Hg})$ & $51(42-64)$ & $40(38-58)$ & $49(34-61)$ \\
\hline RQ & $0.90 \pm 0.01$ & $0.89 \pm 0.02$ & $0.89 \pm 0.02+$ \\
\hline $\mathrm{VO}_{2}(\mathrm{~mL} / \mathrm{kg}$ per min $)$ & $5.3 \pm 1.0$ & $6.5 \pm 1.5$ & $6.6 \pm 2.2+$ \\
\hline
\end{tabular}

For these measurements, expired air escaping from the expiratory valve and via mouth and nose was sampled simultaneously and the measurements are, therefore, not affected by tube leaks. Transcutaneously measured $\mathrm{PCO}_{2}\left(\mathrm{Tcpco}_{2}\right)$ was not different at start and end of the measurements.

$\S$ mean $\pm \mathrm{SD}$.

* median (range).

+ seven preterm infants on nasal CPAP in room air.

\section{Validation during Intermittent Positive Pressure Ventilation}

A calorimetry system for ventilated preterm infants should be compatible with constant-flow pressure-controlled ventilators because these are routinely used in preterm infants. We validated our breath collector in vitro in combination with two mechanical ventilators for neonates.

If one uses the Deltatrac indirect calorimeter as was used in this study or another metabolic cart with a constant flow, the flow to the calorimeter must be higher than the flow from the ventilator to ensure that no expired air is lost. We demonstrated that gas sampling with our collector was complete with a calorimeter flow of $6 \mathrm{~L} / \mathrm{min}$ and a ventilator flow of $4 \mathrm{~L} / \mathrm{min}$, which was the minimal flow required to reach PIP up to $30 \mathrm{~cm}$ $\mathrm{H}_{2} \mathrm{O}$ during short inspiratory times $(0.3 \mathrm{~s})$. In a previous animal study, gas sampling was complete when the internal constant flow of the calorimeter was at least $10 \%$ higher than the ventilator flow (16). Lucas et al. (17) achieved accurate measurements of $\mathrm{Vco}_{2}$ in preterm infants by adjusting calorimeter flow approximately $25 \%$ higher than ventilator flow.

Our measurements with the breath collector were accurate independent of the PIP. We could not test pressures $>24 \mathrm{~cm}$ $\mathrm{H}_{2} \mathrm{O}$ in vitro because then pressure impeded methanol burning.

The response time of our system to a step change in methanol delivery was only $2 \mathrm{~min}$ because the breath collector had a volume of only $200 \mathrm{~mL}$. Lucas et al. (17) used a 6-8-L plastic bag to collect the gases expelled from the ventilator but did not report the response time.

Our breath collector should also be compatible with volumecontrolled ventilators, though we did not validate this. When indirect calorimetry is performed in ventilated infants, the type of ventilator and the type of calorimeter used should be reported together with detailed validation data for this combination of instruments.

\section{The Tube Leak}

A calorimetry system for ventilated preterm infants should capture the expired gases bypassing the endotracheal tube (tube leak). Tube leaks occur frequently in preterm infants $(4,14)$ because uncuffed endotracheal tubes are routinely used and result in an underestimation of $\mathrm{Vo}_{2}$ and $\mathrm{VcO}_{2}$.

Our collector simultaneously and continuously collected air escaping via the tube leak through the infant's mouth and nose and air coming out of the expiratory valve of the ventilator. Thus, the expired air was always sampled completely, regardless of the size of the tube leak and even when a complete tube leak was simulated. Simultaneous sampling of air from the ventilator and from the patient's mouth and nose has been described by Selby et al. (18) for ventilated children using a large hood, but they did not provide in vitro validation data. In our clinical measurements, we frequently detected tube leaks, and they were highly variable between infants.

Other methods proposed to correct for the tube leak have relevant drawbacks. In research settings, the tube leak was determined by directly measuring the infant's inspired and expired flow rates $(14,16)$, by measuring $\mathrm{CO}_{2}$ over the infant's face by infrared capnometry $(4,10)$, or by using double-circuit calorimetry (19). These methods require use of additional pneumotachographs, lung function monitors, or $\mathrm{CO}_{2}$ analyzers and complicate the measurement setup and data analysis. Measuring the tube leak only at the beginning and end of the actual measurement $(13,17)$ cannot provide continuous data 
for the total measurement period. If all infants with audible air leaks were excluded from analysis as was done in clinical studies of EE $(5,20)$, up to $50 \%$ of otherwise eligible infants could not be studied (21) and, even without an audible tube leak, up to $8 \%$ of exhaled breath bypassed the uncuffed endotracheal tube (14).

\section{Nasal CPAP}

A calorimetry system for ventilated preterm infants should be able to measure infants on nasal CPAP, because this ventilatory support is frequently and increasingly used in preterm infants $(22,23)$. Nasal CPAP produced a maximal tube leak, but still up to $16 \%$ of the expired air came from the ventilator outlet. Thus, only a system that continuously and simultaneously samples air from the infant's mouth and nose and from the ventilator, like our collector, can be used in preterm infants on nasal CPAP.

\section{Measurements in Increased Fio 2}

A calorimetry system for ventilated preterm infants should be able to measure EE in infants requiring increased $\mathrm{Fio}_{2}$. We did not perform an in vitro validation of our system in increased $\mathrm{FiO}_{2}$ because of the risk of methanol explosion. Yet, the calorimeter we used has been extensively validated in vitro in raised $\mathrm{Fio}_{2}(6,24,25)$, and accuracy was acceptable up to an $\mathrm{FiO}_{2}$ of 0.6 . With a calorimeter producing a constant internal flow, continuous $\mathrm{Vo}_{2}$ measurements at raised $\mathrm{Fio}_{2}$ require considerable technical effort (19). Therefore, instead of measuring $\mathrm{Vo}_{2}$ directly, we calculated $\mathrm{Vo}_{2}$ from the continuously measured $\mathrm{Vco}_{2}$ and a short-term RQ measurement (18) in intubated preterm infants at raised $\mathrm{Fio}_{2}$, with the assumption that no relevant RQ changes occurred during the total measurement period. The RQ measurement required reducing the calorimeter flow and connecting the respirator to an oxygen tank because this minimizes Fio $_{2}$ fluctuations (14) that disturb the measurements (26).

During nasal CPAP at raised $\mathrm{Fio}_{2}$, we could only measure $\mathrm{Vco}_{2}$. EE can be calculated from $\mathrm{Vco}_{2}$ with a fixed RQ estimate (26) or an RQ estimation from nutritional intake (27). Both RQ estimates have been used in preterm infants to calculate EE from $\mathrm{Vco}_{2}$ measured by the different disappearance rates of stable water isotopes (doubly labeled water method) $(28,29)$.

\section{Use in the Clinical Setting}

A calorimetry system for ventilated preterm infants should be easy to use and should not irritate the patient. Our calorimetry system can be used at the bedside in the intensive care unit and does not require transfer of the infant to a metabolic laboratory or to a research incubator. Connecting the breath collector to the ventilator required no or only a short disconnection of the ventilator circuit. We used the system only with nasotracheally intubated infants, but it can also be used with orotracheal tubes.

We have previously shown that the collection of exhaled gas with a sampling device held close to the infant's face but without actually touching it does not result in increased unrest or changes in body temperature (8). During the present measurements, the infants were sleeping $85 \%$ of the time.

A handheld device like ours requires the continuous presence of a research assistant at the bedside. Measurements over 4-6 h, necessary to extrapolate to a $24-\mathrm{h}$ EE, are possible with a handheld device but no continuous measurements over $24 \mathrm{~h}$. Using a hood is no alternative in very-low-birth-weight infants because for accurate sampling of expired air, high flows through the hood are necessary that lead to excessive dilution of expired air (8).

In conclusion, we have validated a breath collector in vitro and have shown its practicability for measurements of $\mathrm{EE}$ in ventilated preterm infants. The simultaneous sampling of gas coming from the ventilator and from the infant's nose and mouth allows precise measurements regardless of tube leak and during nasal CPAP. Therefore, our system improves the use of indirect calorimetry in ventilated preterm infants and provides an accurate tool for research in energy metabolism in these critically ill patients.

\section{REFERENCES}

1. Grant J, Denne SC 1991 Effect of intermittent versus continuous enteral feeding on energy expenditure in premature infants. J Pediatr 118:928-932

2. Bauer K, Cowett RM, Howard GM, van Epp J, Oh W 1993 Effect of intrauterine growth retardation on postnatal weight change in preterm infants. J Pediatr 123:301306

3. Wahlig TM, Georgieff MK 1995 The effects of illness on neonatal metabolism and nutritional management. Clin Perinatol 22:77-96

4. Knauth A, Baumgart S 1990 Accurate, noninvasive quantitation of expiratory gas leak from uncuffed infant endotracheal tubes. Pediatr Pulmonol 9:55-60

5. Wahlig TM, Gatto CW, Boros SJ, Mammel MC, Mills MM, Georgieff MK 1994 Metabolic response of preterm infants to variable degrees of respiratory illness. J Pediatr 124:283-288

6. Schulze A, Whyte RK, Way RC, Sinclair JC 1995 Effect of the arterial oxygenation level on cardiac output, oxygen extraction, and oxygen consumption in low birth weight infants receiving mechanical ventilation. J Pediatr 126:777-784

7. Takala J, Keinänen O, Väisänen P, Kari A 1989 Measurement of gas exchange in intensive care: laboratory and clinical validation of a new device. Crit Care Med 17:1041-1047

8. Bauer K, Pasel K, Uhrig C, Sperling P, Versmold H 1997 Comparison of face mask, head hood, and canopy for breath sampling in flow-through indirect calorimetry to measure $\mathrm{VO}_{2}$ and $\mathrm{VCO}_{2}$ of preterm infants $<1500 \mathrm{~g}$. Pediatr Res 41:139-144

9. Brueck K, Parmalee Jr AH, Brueck M 1962 Neutral temperature range and range of thermal comfort in premature infants. Biol Neonate 4:32-38

10. Shortland GJ, Fleming PJ, Walter JH 1992 Validation of a portable indirect calorimetry system for measurement of energy expenditure in sick preterm infants. Arch Dis Child 67:1207-1211

11. Rivera A, Bell EF, Bier DM 1993 Effect of intravenous amino acids on protein metabolism of preterm infants during the first three days of life. Pediatr Res 33:106-111

12. Mayfield SR 1991 Technical and clinical testing of a computerized indirect calorimeter for use in mechanically ventilated neonates. Am J Clin Nutr 154:30-34

13. Forsyth JS, Crighton A 1992 An indirect calorimetry system for ventilator dependent very low birthweight infants. Arch Dis Child 67:315-319

14. Thureen PJ, Phillips RE, DeMarie MP, Hoffenberg A, Bronstein MN, Spedale SB, Hay WW 1997 Technical and methodological considerations for performance of indirect calorimetry in ventilated and non-ventilated preterm infants. Crit Care Med 25:171-180

15. Bracco D, Chiolero R, Pasche O, Revelly JP 1995 Failure in measuring gas exchange in the ICU. Chest 107:1406-1410

16. Räsänen J 1992 Continuous breathing circuit flow and tracheal tube cuff leak: sources of error during pediatric indirect calorimetry. Crit Care Med 20:1335-1340

17. Lucas A, Nohria V, Roberts SB 1987 Measurement of carbon dioxide production rate in sick ventilated premature infants. Biol Neonate 51:138-143

18. Selby AM, McCauley JC, Schell DN, O'Connell A, Gillis J, Gaskin KJ 1995 Indirect calorimetry in mechanically ventilated children: a new technique that overcomes the problem of endotracheal tube leak. Crit Care Med 23:365-370

19. Roze JC, Chambille B, Dehan M, Gaultier C 1994 Measurement of oxygen uptake in newborn infants during assisted and spontaneous ventilation. Respir Physiol 98:227239

20. Billeaud C, Piedboeuf B, Chessex P 1992 Energy expenditure and severity of respiratory disease in very low birth weight infants receiving long-term ventilatory support. J Pediatr 120:461-464 
21. Chwals WJ, Lally KP, Woolley MM 1992 Indirect calorimetry in mechanically ventilated infants and children: measurement accuracy with absence of audible air leak. Crit Care Med 20:768-770

22. Higgins RD, Richter SE, Davis J 1991 Nasal continuous positive airway pressure facilitates extubation of very low birth weight neonates. Pediatrics 88:999-1003

23. Kamper J, Wulff K, Larsen C, Lindequist S 1993 Early treatment with nasal continuous positive airway pressure in very low-birth-weight infants. Acta Paediatr 82:193-197

24. Weissman C, Sardar A, Kemper M 1990 In vitro evaluation of a compact metabolic measurement instrument. JPEN 14:216-221

25. Weyland W, Weyland A, Fritz U, Redecker K, Ensink FB, Braun U 1994 A new pediatric metabolic monitor. Intensive Care Med 20:51-57
26. Kalhan SC, Denne SC 1990 Energy consumption in infants with bronchopulmonary dysplasia. J Pediatr 116:662-664

27. Black AE, Prentice AM, Coward WA 1986 Use of food quotients to predict respiratory quotients for the doubly labelled water method of measuring energy expenditure. Hum Nutr Clin Nutr 40C:381-391

28. Roberts SB, Coward WA, Schlingenseipen KH, Nohria V, Lucas A 1986 Comparison of the doubly labelled water $\left({ }^{2} \mathrm{H}_{2}{ }^{18} \mathrm{O}\right)$ method with indirect calorimetry and a nutrient balance study for simultaneous determination of energy expenditure, water intake, and metabolizable energy intake in preterm infants. Am J Clin Nutr 44:315-322

29. Jensen CL, Butte NF, Wong WW, Moon JK 1992 Determining energy expenditure in preterm infants: comparison of ${ }^{2} \mathrm{H}_{2}{ }^{18} \mathrm{O}$ method and indirect calorimetry. Am J Physiol 263:R685-R692 